\title{
MicroRNA markers for forensic body fluid identification obtained from microarray screening and quantitative RT-PCR confirmation
}

\author{
Dmitry Zubakov • Anton W. M. Boersma • Ying Choi • \\ Patricia F. van Kuijk • Erik A. C. Wiemer • \\ Manfred Kayser
}

Received: 16 October 2009 /Accepted: 10 December 2009/Published online: 10 February 2010

(C) The Author(s) 2010. This article is published with open access at Springerlink.com

\begin{abstract}
MicroRNAs (miRNAs) are non-protein coding molecules with important regulatory functions; many have tissue-specific expression patterns. Their very small size in principle makes them less prone to degradation processes, unlike messenger RNAs (mRNAs), which were previously proposed as molecular tools for forensic body fluid identification. To identify suitable miRNA markers for forensic body fluid identification, we first screened total RNA samples derived from saliva, semen, vaginal secretion, and venous and menstrual blood for the expression of 718 human miRNAs using a microarray platform. All body fluids could be easily distinguished from each other on the basis of complete array-based miRNA expression profiles. Results from quantitative reverse transcription PCR (RTPCR; TaqMan) assays for microarray candidate markers confirmed strong over-expression in the targeting body fluid of several miRNAs for venous blood and several others for semen. However, no candidate markers from array experiments for other body fluids such as saliva, vaginal secretion, or menstrual blood could be confirmed by RT-PCR. Time-wise degradation of venous blood and semen stains for at least 1 year under lab conditions did not significantly affect the detection sensitivity of the identified
\end{abstract}

Electronic supplementary material The online version of this article (doi:10.1007/s00414-009-0402-3) contains supplementary material, which is available to authorized users.

D. Zubakov $\cdot$ Y. Choi $\cdot$ M. Kayser $(\triangle)$

Department of Forensic Molecular Biology,

Erasmus University Medical Center Rotterdam,

Rotterdam, The Netherlands

e-mail: m.kayser@erasmusmc.nl

A. W. M. Boersma - P. F. van Kuijk • E. A. C. Wiemer

Department of Medical Oncology, Josephine Nefkens Institute, Erasmus University Medical Center Rotterdam,

Rotterdam, The Netherlands
miRNA markers. The detection limit of the TaqMan assays tested for selected venous blood and semen miRNA markers required only subpicogram amounts of total RNA per single RT-PCR test, which is considerably less than usually needed for reliable mRNA RT-PCR detection. We therefore propose the application of several stable miRNA markers for the forensic identification of blood stains and several others for semen stain identification, using commercially available TaqMan assays. Additional work remains necessary in search for suitable miRNA markers for other forensically relevant body fluids.

Keywords miRNA - Body fluid identification · Microarray . TaqMan $\cdot$ RT-PCR $\cdot$ Forensics

\section{Introduction}

MicroRNAs (miRNAs) belong to a class of small nonprotein coding RNA molecules of 18 to 22 nucleotides in length that negatively regulate gene expression. Associated with a multiprotein complex, the RNA-induced silencing complex (RISC) miRNAs hybridize to the $3^{\prime}$ UTR of specific messenger RNA (mRNA) targets, causing inhibition of mRNA translation and/or mRNA degradation [1]. A number of miRNAs have been implicated in the processes of embryonic development, cell proliferation, and differentiation, as well as in the pathogenesis of many human diseases such as cancer and neurodegenerative and metabolic disorders [1,2]. Taking into account the involvement of miRNAs in developmental processes, it is not surprising that several recent studies revealed tissue-specific expression patterns of many miRNAs $[3,4]$. Correctly identifying body fluids in forensic practice is important for crime scene reconstruction, and commonly used protein-based assays 
are prone to various limitations $[5,6]$. Recently, mRNA profiling has been proposed as a sensitive and specific method for forensic applications, particularly the use of mRNAs with tissue-specific expression for body fluid identification [7, 8]. It has been shown that several mRNA markers with tissue-specific expression pattern can be detected in biological stains after long periods of storage under dry, sun-protected, and ambient-temperature conditions, making them potentially useful for body fluid identification in forensic practice [9]. However, mRNA degradation is expected to be greatly promoted by aggressive environmental factors (e.g., UV light, moisture, and heat); all can be relevant for forensic samples. Since physical fragmentation of RNA molecules due to degradation dictates the use of very short amplicons for successful PCR detection, the intrinsically short miRNAs hold promises for being ideal markers for forensic applications and in case of significant differential expression, for forensic body fluid identification. The number of identified miRNAs is constantly growing, currently reaching many hundreds of species for humans. Such number requires the use of high-throughput methods, such as microarrays, for effective miRNA candidate screening. Here, we applied custom microarrays using $\mathrm{LNA}^{\mathrm{TM}}$ modified oligonucleotides as capture probes for 718 known human miRNAs to samples from five forensically relevant body fluids of several individuals. Candidate miRNA markers identified from microarray data were validated using TaqManbased reverse transcription PCR (RT-PCR) technology and Northern blotting, thus providing robust confirmation of differentially expressed miRNA markers with different methods. MiRNA markers with confirmed differential expression were further tested for degradation stability, and additionally, the sensitivity of marker-specific TaqMan assays was assessed.

\section{Materials and methods}

\section{Body fluid samples and RNA extraction}

Body fluids, i.e., saliva, venous blood, menstrual blood, semen, and vaginal secretion, were collected from three male and three female volunteers with informed consent. For saliva collection, volunteers were asked to avoid eating and drinking for at least half an hour before sampling, and each unstimulated $2 \mathrm{~mL}$ saliva specimen was expectorated into a 50-mL Falcon tube placed on ice and immediately processed for RNA isolation. Venous blood samples were obtained by vein puncture using dry Vaccutainer tubes; within minutes, before coagulation occurred, $1 \mathrm{~mL}$ of blood was added to RNA extraction buffer. Menstrual blood and vaginal secretion samples were obtained using overnight tampons. Semen samples were collected by ejaculation in 50-mL Falcon tubes, left for $\sim 5$ min at RT until liquefaction of the ejaculate, and 1-mL aliquots were added to RNA extraction buffer supplemented with $50 \mathrm{mM} \beta$-mercaptoethanol to ensure complete lysis of the sperm cells. Time-wise-degraded series of samples were obtained by storage of either cotton swabs (blood, saliva, and semen) or tampons (menstrual blood and vaginal secretion) at ambient dust-free lab conditions (no UV exposure, artificial light exposure, limited day light exposure, and room temperature and room humidity without significant variations) for a period of up to 1 year. Total RNA extraction was performed using the RNA-Bee reagent (Bio-Connect, Huissen, The Netherlands) following the instructions of the manufacturer. Potential traces of genomic DNA were removed with DNase I digestion performed with Ambion's Turbo DNA-free kit (Applied Biosystems, Nieuwerkerk aan den IJssel, The Netherlands) according to manufacturer's protocol. The purity and quantity of RNA were assessed with a NanoDrop ND-1000 spectrophotometer (Thermo Scientific, Wilmington, DE, USA).

\section{Microarray analysis}

MiRNA profiling experiments using LNA ${ }^{\mathrm{TM}}$-modified oligonucleotides (Exiqon, Vedbæk, Denmark) as capture probes were essentially performed as described by Pothof et al. [10] with the exception that the capture probes used here were based on version 10.1 of the miRNA registry (http://www. mirbase.org). The arrays were capable of detecting the expression levels of 718 human miRNAs as well as the full complement of rodent miRNAs, various additional nonprotein coding RNAs, and some viral miRNAs. The hybridization signals of 2,688 spots were normalized using quantile algorithm. Data points with low signal intensities $(<10)$ in all the samples were excluded from further analysis. Hierarchical clustering, significance analysis of microarrays (SAM), and one-way analysis of variance (ANOVA) were performed using TM4 software [11]. The subsets of miRNAs that best characterize each body fluid were identified using nearest shrunken centroids method implemented in prediction analysis of microarrays (PAM) software [12]. The selection of differentially expressed miRNA markers for subsequent confirmation via quantitative RT-PCR analysis was mainly based on PAM scores, but also invoked the fold-change of differential expression and absolute expression levels. Preference was given to markers that are abundant in the target body fluids, but are only minimally expressed in the non-target ones.

\section{TaqMan RT-PCR}

Validation of the expression of selected miRNA markers was performed using commercially available pre-designed TaqMan RT-PCR assays (Applied Biosystems, Nieuwer- 
kerk aan den IJssel, The Netherlands) (see Electronic supplementary materials, Table 1 for details) using the very same RNA samples that were used for the microarray profiling. The TaqMan MicroRNA Reverse Transcription Kit (Applied Biosystems) was used for the preparation of cDNA. Reverse transcription reactions were performed in a volume of $15 \mu \mathrm{L}$, and each reaction contained $100 \mathrm{ng}$ of total RNA and either single or multiple (pentaplex) stemloop miRNA-specific primers in experiments of fresh and time-wise-degraded samples. Two microliters of a 2.5 -fold diluted cDNA mix was used as input in each individual PCR. For the assay sensitivity test, a 10-fold dilution series of total RNA was used, ranging from 20 to $0.002 \mathrm{ng}$ per pentaplex RT reaction. All TaqMan assays were run in triplicate on an ABI Prism 7300 using TaqMan universal PCR master mix without UNG (Applied Biosystems) and applying 45 PCR cycles. Amplification signals were computed with the SDS v.2.1 software (Applied Biosystems). Relative quantification of miRNA expression was performed by the delta $\mathrm{Ct}(\mathrm{dCt})$ method using the average expression of three reference control genes (small RNAs RNU24, RNU44, and RNU48 analyzed by commercially available TaqMan assays) for normalization of miRNA abundances across the tissues. The expression levels for each marker were converted in a linear scale as fold-change (FC) values calculated by the formula: $\mathrm{FC}=2 * * \mathrm{dCt}$. Error propagation formula was used for the calculation of standard deviations (SD) of the average fold-change of expression: $\mathrm{SD}_{\mathrm{FC}}=\operatorname{Ln}(2) * \mathrm{SD}_{\mathrm{dCt}} * \mathrm{FC}$, where $\mathrm{SD}_{\mathrm{dCt}}=\mathrm{SQRT}\left(\mathrm{SD}_{\text {target Ct }} * * 2+\mathrm{SD}_{\text {reference Ct }} * 2\right) / \mathrm{SQRT}(\mathrm{n})$, $\mathrm{n}=$ number of replicates (normally 3 ).

\section{Northern blotting}

Total RNA was isolated from two semen and two vaginal secretion samples with TRIzol reagent (Invitrogen, Carlsbad, CA, USA). Ten micrograms of RNA per sample was fractionated on a denaturing $15 \%$ polyacrylamide gel along with an Ultra Low-Range Gene-Ruler DNA ladder (Fermentas AG, Germany). After the gel run, the size ladder was cut from the polyacrylamide gel and stained with ethidium bromide and photographed for future determination of the size of hybridized fragments. RNA present in the remainder of the gel was transferred to a nylon membrane (Hybond N+; Amersham Pharmacia Biotech, Rosendaal, The Netherlands) by electroblotting. After being UV-crosslinked $(120 \mathrm{~mJ})$ and baked at $80^{\circ} \mathrm{C}$ for $30 \mathrm{~min}$, the membrane was hybridized with an miR-891a LNA probe (Exiqon A/S, Vedbæk, Denmark) at $65^{\circ} \mathrm{C}$ overnight in PerfectHyb Plus hybridization buffer (Sigma-Aldrich, The Netherlands). Hybridization signals were detected with chemiluminescent NEB Phototope ${ }^{\circledR}$-Star Detection Kit (Westburg BV, Leusden, The Netherlands) according to the manufacturer's instructions. Blots were scanned with
Typhoon 9410 system (GE Healthcare, Brussels, Belgium). Equal amounts of total RNA in each sample served as an internal loading control.

\section{Results and discussion}

Microarray data analysis

After filtering out the low-intensity hybridization signals across all body fluid and individual samples, 458 of 718 $(63.8 \%)$ miRNAs remained in the dataset and were used for further analysis. Unsupervised hierarchical clustering of normalized hybridization values revealed that samples belonging to the same body fluid tend to cluster together (Fig. 1) and that different body fluids display distinct miRNA expression signatures. Hence, based on the microarray results, all five body fluids could be clearly differentiated from each other based on miRNA expression. SAM and ANOVA analyses provided largely identical sets of differentially expressed miRNAs (results not shown). In search for the most informative miRNA markers, the PAM algorithm was employed and identified a total subset of 294 candidate markers that best characterized the five body fluids in the microarray dataset (see Electronic supplementary materials, Fig. 1). Further manual ascertainment of the most promising candidate markers from this list was performed considering the fold change of differential expression and absolute expression levels, resulting in small candidate markers sets for each tissue for validation by quantitative RT-PCR.

\section{Validation of candidate miRNA markers}

A total of 14 most promising candidate markers (two to four for each of the five body fluids) were selected from the microarray dataset for validation in TaqMan-based quantitative RT-PCR assays (Table 1). This step was important not only as technical confirmation of the microarray results using an independent methodology but also because RT-PCR assays, instead of microarray profiling, can easily be applied to forensic samples. All ascertained candidate miRNAs could be detected with commercially available TaqMan assays, irrespective of whether multiplex (pentaplex) or singleplex cDNA synthesis reactions were performed. Three small RNAs, RNU24, RNU44, and RNU48 used as endogenous reference controls, demonstrated similar levels of expression within each sample and irrespective of the sample origin.

The level of agreement between the microarray and RTPCR (TaqMan) expression profiling results was highly dependent on the body fluid in focus. In particular, the expression patterns of semen and venous blood-targeting candidate markers were highly concordant between both 
Fig. 1 Unsupervised hierarchical clustering of 458 different miRNAs from microarray analysis of five forensically relevant body fluids, i.e., semen $(S E)$, saliva $(S A)$, vaginal secretion $(V S)$, menstrual blood $(M S)$, and whole venous blood $(W B)$ from several individuals. Note the clustering of samples according to body fluids based on the miRNA expression

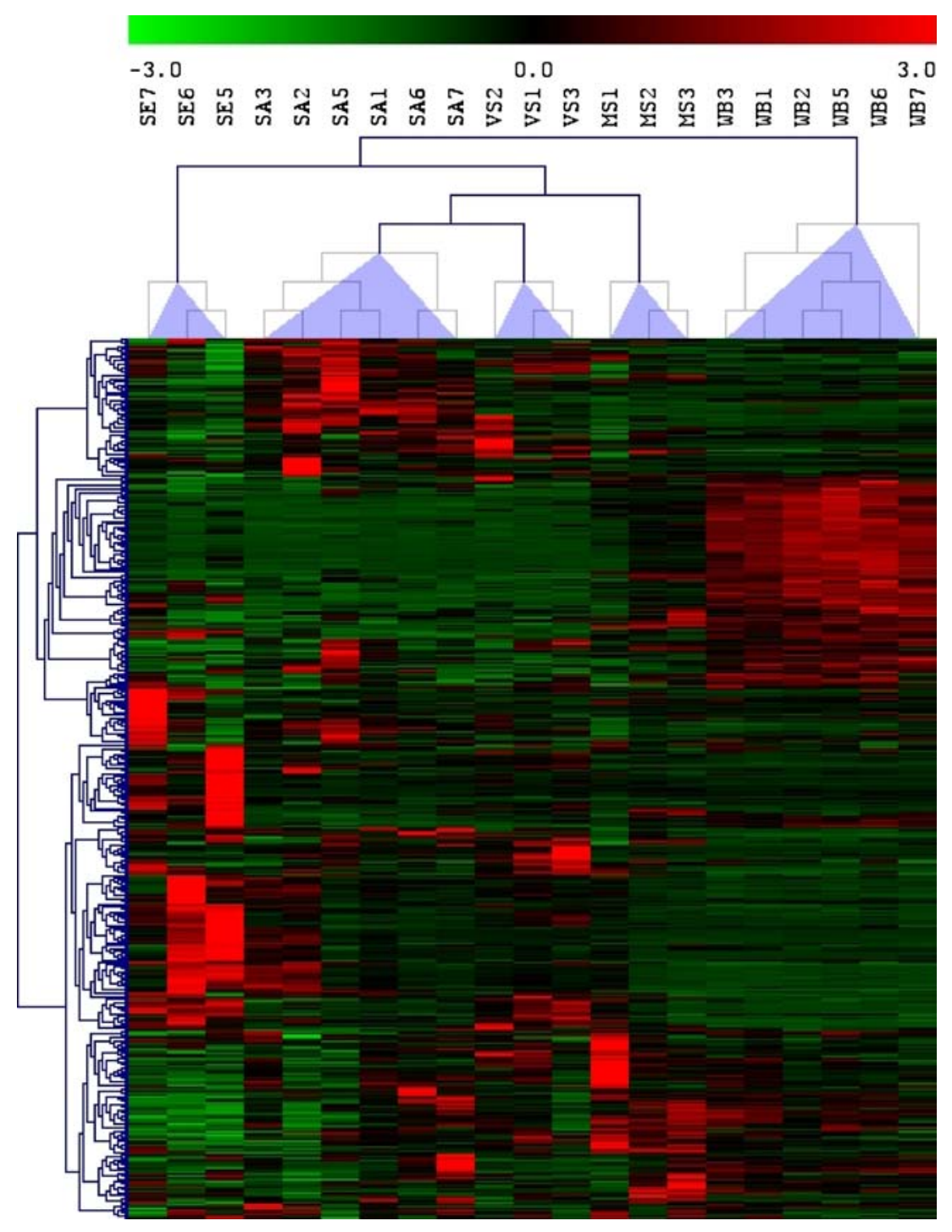

detection methodologies. Both the microarray and the RTPCR (TaqMan) analyses confirm the over-expression of candidate miRNAs in the target body fluids and show an overall high correlation between microarray and RT-PCR results (Fig. 2). Three miRNAs, miR-20a, miR-106a, and miR-185, revealed high expression levels in venous blood relative to all other body fluids tested, including menstrual blood and with agreeing results from both technologies (except for a single individual menstrual blood sample where miR-185 expression was in the range of venous blood). Furthermore, four miRNAs, miR-135a, miR-10a,
miR-507, and miR-943, appeared strongly over-expressed in semen relative to all other body fluids tested and with agreeing results from both technologies. However, less concordance between microarray and RT-PCR results was achieved for the other three body fluids (Electronic supplementary materials, Fig. 2). In particular, TaqMan analysis of the microarray candidate markers for menstrual blood revealed that miR-144 was down-regulated in semen, saliva, and vaginal secretion, in agreement with the microarray results. However, miR-144 expression from TaqMan analysis appeared more abundant in venous blood than in
Table 1 Candidate miRNA markers with differential expression patterns in five body fluids as derived from microarray data analysis and used for RT-PCR confirmation analysis

\begin{tabular}{lllll}
\hline Menstrual blood & Venous blood & Semen & Saliva & Vaginal secretion \\
\hline hsa-miR-185* & hsa-miR-20a & hsa-miR-943 & hsa-miR-583 & hsa-miR-617 \\
hsa-miR-144 & hsa-miR-106a & hsa-miR-135a & hsa-miR-518c* & hsa-miR-891a \\
& hsa-miR-185 & hsa-miR-10a & hsa-miR-208b & \\
& & hsa-miR-507 & & \\
& & & \\
\hline
\end{tabular}


menstrual blood in disagreement with the microarray data (Fig. 2a). Hence, our RT-PCR data suggest that miR-144 is a useful marker for all kinds of blood samples (including menstrual blood), but this miRNA was not confirmed as specific menstrual blood marker. Another microarray candidate marker for menstrual blood, miR-185*, showed a non-specific expression across all body fluids in the RTPCR results not agreeing with the microarray results (Electronic supplementary materials, Fig. 2). Also the miRNAs, miR-583, miR-518c*, and miR-208b identified by microarray analysis as candidate markers for saliva and miR-617 for vaginal secretion, demonstrated a strong discordance between microarray and TaqMan data (Electronic supplementary materials, Fig. 2). None of these markers displayed the expected expression pattern when assayed by RT-PCR. In an attempt to find specific markers for saliva, vaginal secretion, and menstrual blood, one additional miRNA candidate marker per each tissue was ascertained from the microarray data, i.e., miR-9*, miR203, and miR-296*, respectively. The usefulness of these additional markers was examined in a limited number of individual samples (e.g., one to two per tissue). However, no over-expression in the targeted body fluids was observed for any of these additional markers (results not shown).

Recently, a set of nine miRNA markers with differential expression in the same five body fluids studied here was reported by Hanson et al. [13]. Except for two semen markers, miR-135b and miR-10b, which are closely related to (but not identical with) miR-135a and miR-10a identified in our screen, there was no overlap with the 14 candidate miRNAs selected by us. Given our problems in identifying reliable miRNA markers for vaginal secretion, menstrual blood, and saliva, we tested the expression of the seven markers from Hanson et al. that were unrelated to our candidate markers, by quantitative RT-PCR (TaqMan) in our sample set (Electronic supplementary materials, Fig. 3). We were only able to replicate the Hanson et al. results for markers these authors suggested for venous blood (miR-16 and miR-451) and semen (miR-10b and miR-135b), but not for those they suggested for saliva (miR-205 and miR-658), vaginal secretion (miR-124a and miR-372), and menstrual blood (miR-412). There may be several explanations for the observed discrepancies between our and the published findings for the latter three body fluids. First, we used TaqMan RT-PCR technology from Applied Biosystems, whereas Hanson et al. utilized SYBR Green assays from Qiagen, which implies different strategies for both cDNA synthesis and PCR quantification. Second, the relatively small sample size, especially in our study, does not allow ruling out the natural variation of miRNA expression that may exist between individuals. However, it did not escape our attention that the two body fluids, i.e., venous blood and semen, for which we could successfully identify and validate specific miRNA markers, are the same two body fluids for which we could replicate the findings of Hanson et al. Conversely, the three body fluids (saliva, vaginal secretion, and menstrual blood) for which we were unable to reliably identify specific miRNA markers in our own screening experiments could also not be identified on the basis of the miRNA markers proposed by Hanson et al. (see below for further explanation).

Notably, the most striking case of disagreement between our microarray and RT-PCR data was observed for miR-891a. According to our microarray data, this particular miRNA appeared to be highly over-expressed in vaginal secretion, whereas our quantitative RT-PCR (TaqMan) assay demonstrated that it was highly and exclusively expressed in semen (Fig. 3a). A literature search revealed, according to one of the large-scale screening studies [14], that this miRNA is present only in epididymis tissue (i.e., part of testicular system where sperm cells maturate) and was practically absent from any other tissue analyzed. This suggested that our microarray data regarding miR-891a possibly reflect an artifact. To test this assumption, we analyzed total RNA from both semen and vaginal secretion by Northern blotting using a biotinylated LNA ${ }^{\mathrm{TM}}$ probe targeting miR-891a. The presence of an RNA species of the expected size of 22 bases in the semen samples (highlighted by circle in Fig. 3b) unequivocally showed that miR-891a is indeed expressed in semen. No hybridization signal of a RNA species with expected fragment size was observed in the vaginal secretion samples (Fig. 3b). Consequently, we established miR-891a as marker highly over-expressed in semen. Moreover, as seen on the Northern blot, the lanes containing the vaginal secretion samples showed a very strong non-miRNA hybridization signal in the high molecular weight area (highlighted by rectangle in Fig. 3b). This may indicate that the vaginal secretion samples contained RNA species that crosshybridize with the miR-891a probe, thereby explaining the erroneous result for miR-891a expression in the microarray analysis. The LNA ${ }^{\mathrm{TM}}$ - modified oligonucleotide miRNA capture probes are designed not to cross-hybridize with other human RNA species (Exiqon, Vadbæk, Denmark). Therefore, one may assume that the cross-hybridizing bands on the Northern blots in the vaginal secretion samples are not derived from the human transcriptome, but perhaps from bacterial and/or fungal RNA, which is expected to be present in total RNA extracts from vaginal samples. This would explain why our microarray experiments failed to identify specific miRNA markers in vaginal secretion. Moreover, the same explanation may apply to saliva and menstrual blood and our inability to identify reliable miRNA markers, since also these body fluids are naturally contaminated with bacteria and fungi. An alternative explanation for the crosshybridizing bands in the case of miR-891a is that in vaginal samples, mir-891a may not be properly processed to its 
mature form and present only as miRNA precursor (cf. the hybridizing bands of 60-75 bases in one of vaginal samples) or as primary miRNA transcript (cf. the hybridizing bands $>300$ bases in both samples). The LNA ${ }^{\mathrm{TM}}$ capture probes used in microarray and Northern blot hybridization may not be able to discriminate mature and unprocessed miRNA. In contrast, stem-loop primers used at reverse transcription step in RT-PCR TaqMan technology are specific only to short mature miRNA molecules, which also make TaqMan method less affected by potentially contaminating bacterial/ fungal RNA. Future screening for candidate miRNA markers for saliva, vaginal secretion, and menstrual blood employing, e.g., highly specific TaqMan technology from Applied Biosystems, may reveal promising markers.

\section{Time-wise stability of miRNA markers}

The effect of long-term storage on the detection of miRNAs was tested by RT-PCR. We examined the stability of the blood markers miR-185, miR-106a, and miR-144 and the semen markers miR-135a, miR-10a, miR-507, and miR-891a using quantitative RT-PCR (TaqMan) assays. The same blood and semen samples as used in the initial experiments were aged for 1 year under lab conditions (relatively constant humidity and ambient temperature, no UV exposure, dust free, etc.). The comparison of PCR results from fresh and 1-year old samples plotted in parallel (Electronic supplementary materials, Fig. 4) revealed strong similarity in expression profiles and absolute expression levels. It is remarkable that not only miRNAs could be easily detected in experimentally aged body fluid samples but also that the absolute levels do not seem to be diminished in old samples. Thus, in agreement with the expectation based on the very small size of miRNAs, practically no effect of time-wise degradation was detected for the miRNA markers tested. Unfortunately, the amount of RNA available in this experiment was limited and did not allow testing the blood marker miR-20a and the semen marker miR-943. However, based on the results of the seven markers tested and the general assumption that miRNAs should not be sensitive to in vitro degradation processes due to their small size, there is no apparent reason to expect the degradation behavior of these two miRNAs being significantly different. Although the evaluation of the effect of environmental factors other than time (such as humidity, temperature, and UV radiation) on miRNA degradation is yet to be investigated in more detailed validation studies, and the excellent time-wise stability of the miRNA markers tested here holds a great promise for forensic applications.

Sensitivity of miRNA TaqMan assays

We used serial dilutions of total RNA (10-fold dilution from 20 to $0.002 \mathrm{ng}$ ) isolated from fresh venous blood and
Fig. 2 TaqMan RT-PCR expression profiles of candidate miRNAs markers over-expressed in whole venous blood (a) or semen (b) according to microarray analysis. The plots on the left side show RTPCR data from five body fluids (for abbreviations, see the legend to Fig. 1) and several individuals as mean fold-change values of technical replicate reactions $\pm \mathrm{SD}$. The plots on the right side show correlation between microarray expression signals and RT-PCR data for the same miRNA marker

semen samples as input for cDNA synthesis, $1 / 18$ of which was used in single PCR test, to establish the detection sensitivity of TaqMan RT-PCR assays for two blood (miR144 and miR-185) and two semen (miR-135a and miR891a) miRNA markers. These four markers were chosen for sensitivity testing because they appeared most abundant and most differentially expressed according to the TaqMan analyses. Hence, they serve as best miRNA markers for blood and semen from our study and the sensitivity levels of their respective TaqMan assays are of relevance for future forensic applications. Strikingly, all four markers were detectable in the target body fluid using the lowest amount of 2 pg total RNA for cDNA synthesis, thus corresponding to as little as $0.1 \mathrm{pg}$ RNA per single RT-PCR test (Electronic supplementary materials, Fig. 5). Such amount of total RNA corresponds to single cell level and is much lower than usually needed for successful RT-PCR detection of mRNAs [9]. Therefore, we expect the miRNA markers suggested here for blood and semen identification to be useful for most forensic cases. It will be interesting to see in future forensic validation studies how the high assay sensitivities established here from variation of template RNA concentrations translate to variation in stain size including different surface materials.

A different aspect of TaqMan assay sensitivity relevant for forensic applications is species specificity. Although we consider experimental testing something for future forensic validation studies, we searched existing information on the markers studied here by RT-PCR. From the "Assay Index File v8 for miRNA" material available online from the assay manufacturer, only information of two mammal species (mouse and rat) was obtainable for species specificity of the assays used. In addition, we explored the Sanger miRNA database to search for complete homology of the human miRNA marker sequences with any other species and selected results of the most relevant species, chimpanzee, dog, and chicken. As evident from this (limited) combined information (Electronic supplementary materials, Table 1), some miRNA markers appear specific to the human species, whereas others do not. This is not unexpected as in general miRNAs are known to be extremely conservative across species; however, some human miRNA are differed by one or few bases from the closest orthologs, which can be reflected in humanspecific TaqMan assays. Of the four blood markers high- 


\section{a}

\section{Blood markers:}

TaqMan miR-144

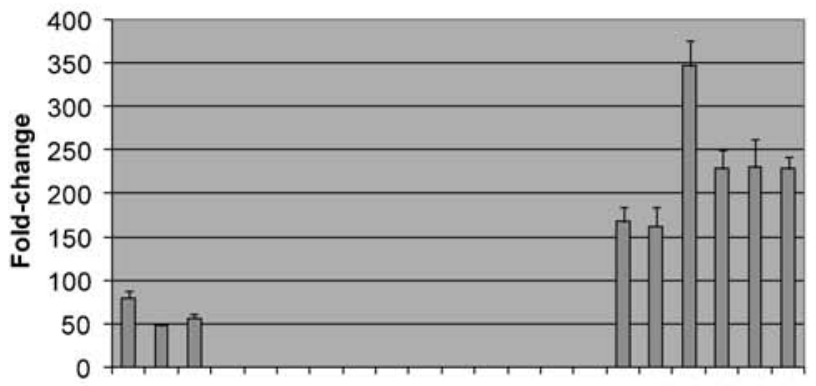

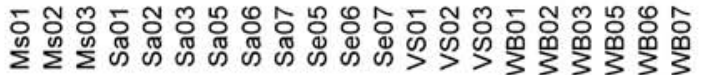

TaqMan miR-20a

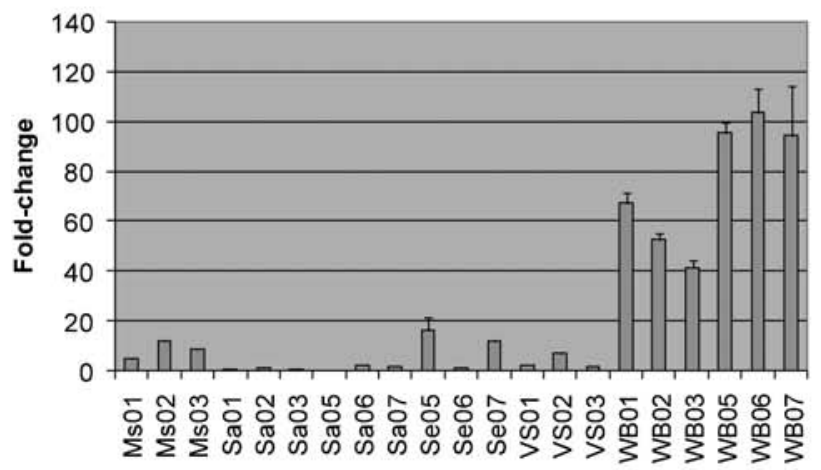

TaqMan miR-185

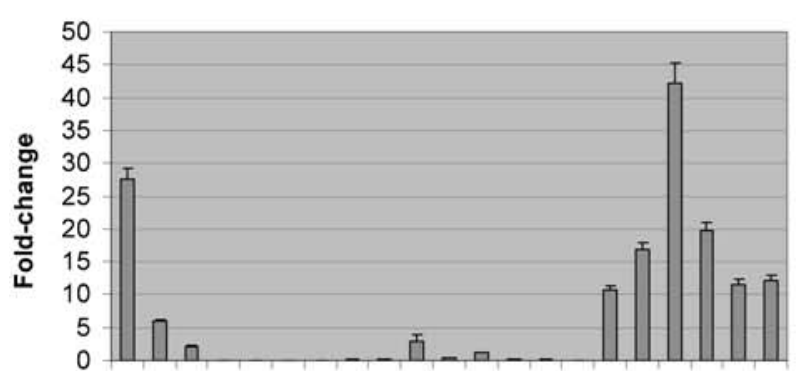

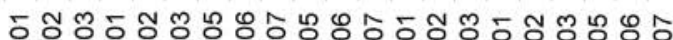

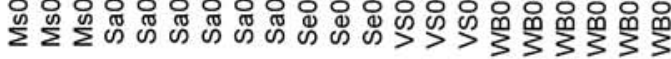

\section{TaqMan miR-106a}

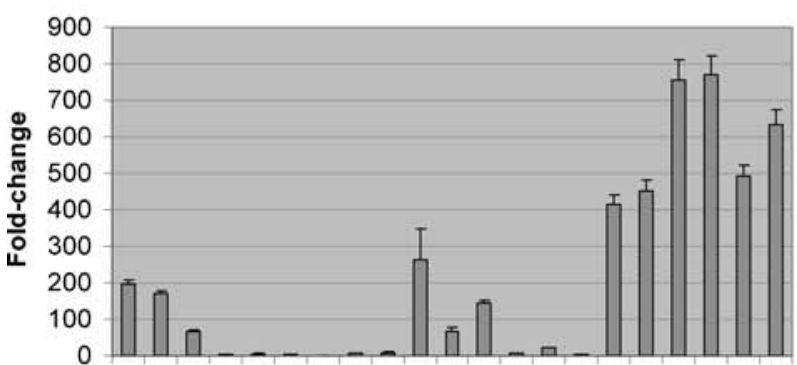

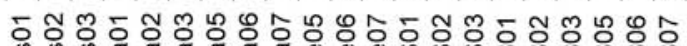

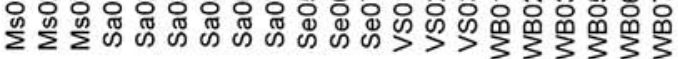
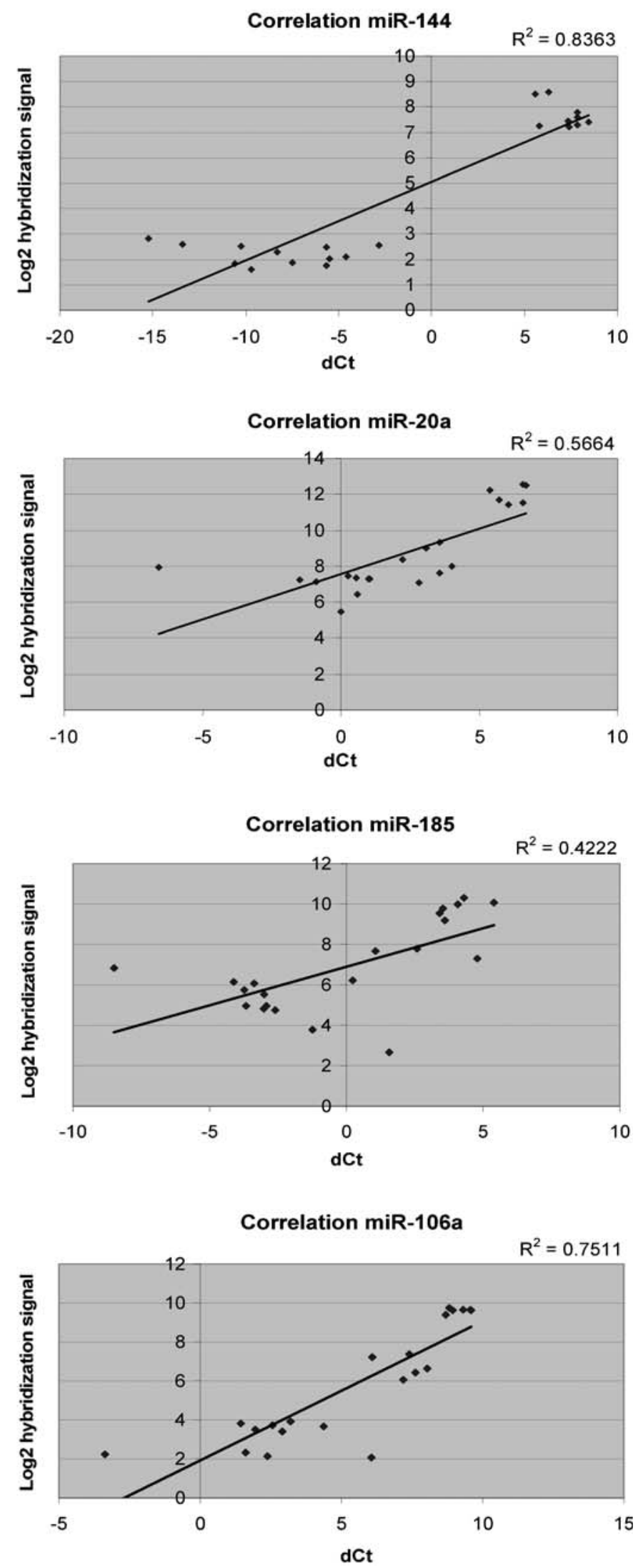
b

\section{Semen markers:}

TaqMan miR-135a

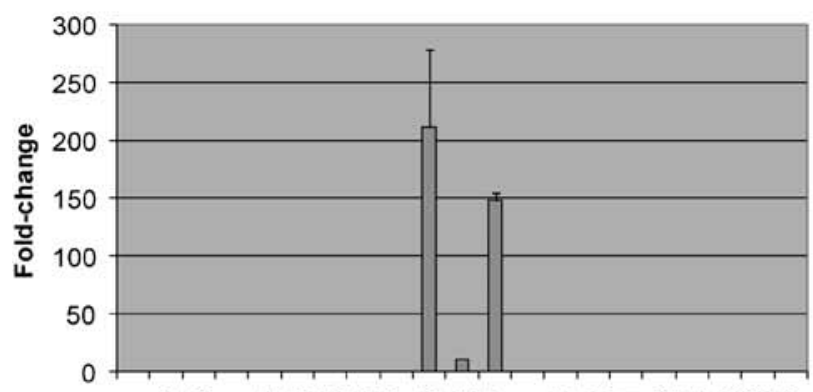

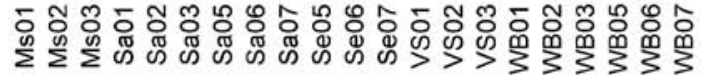

\section{TaqMan miR-943}

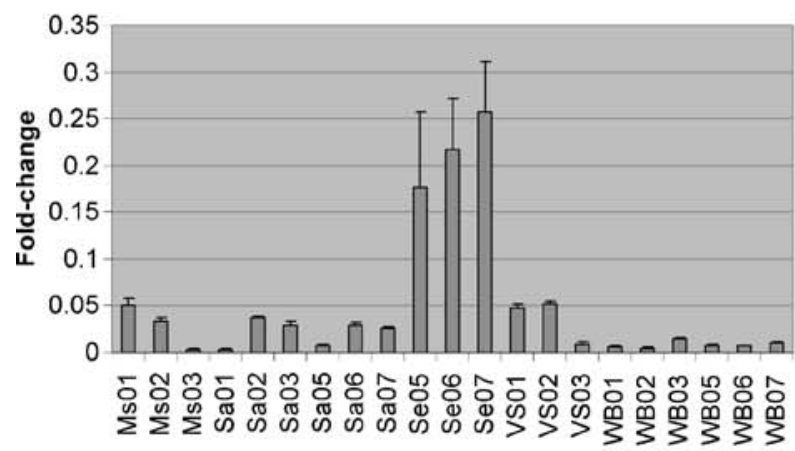

TaqMan miR-10a

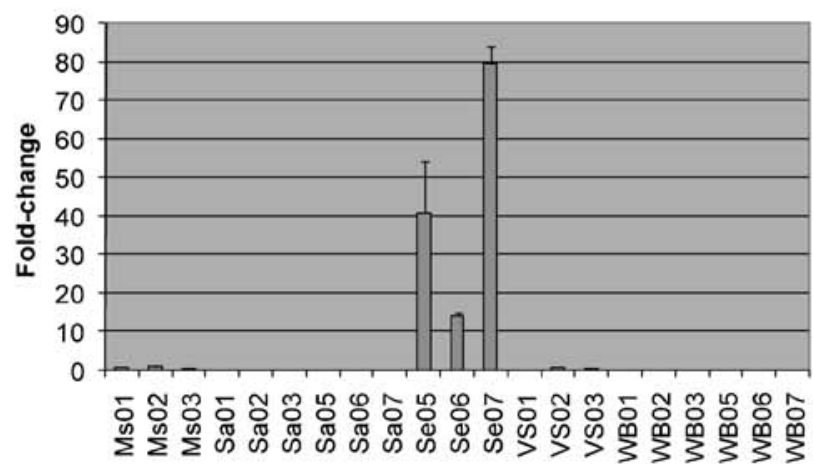

TaqMan miR-507

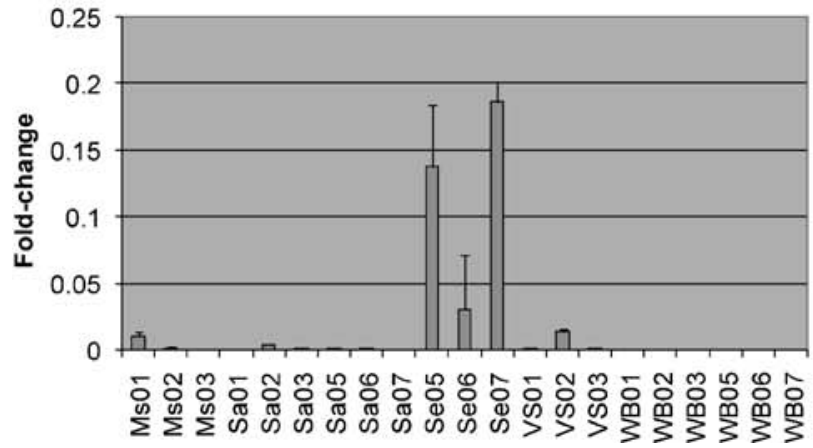

Fig. 2 (continued)
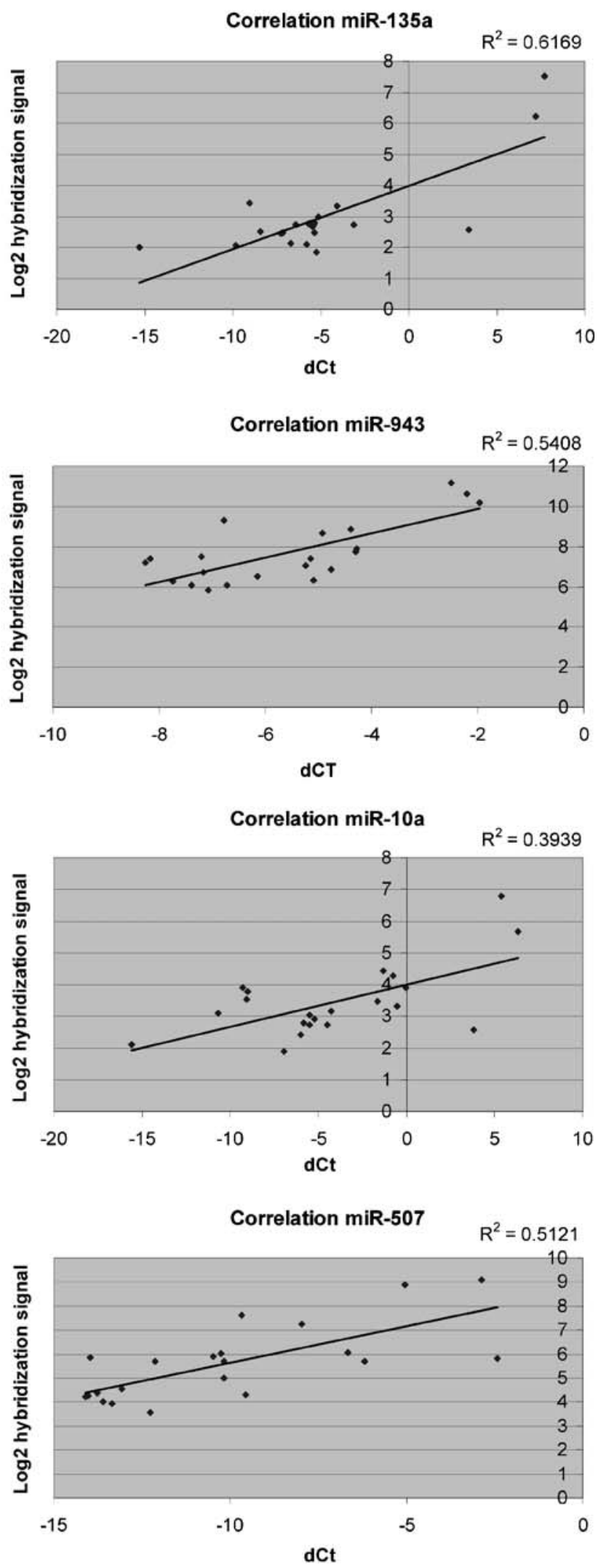
Fig. 3 Expression profile of miRNA marker miR-891a. a Expression of miR-891a according to microarray and TaqMan RT-PCR analyses in five body fluids (for abbreviations, see Fig. 1) from several individuals. b Northern blot of total RNA from semen $(S E)$ and vaginal secretion $(V S)$ samples hybridized with a miR-891a specific LNA ${ }^{\mathrm{TM}}$ oligonucleotide ( $L$ size ladder) a

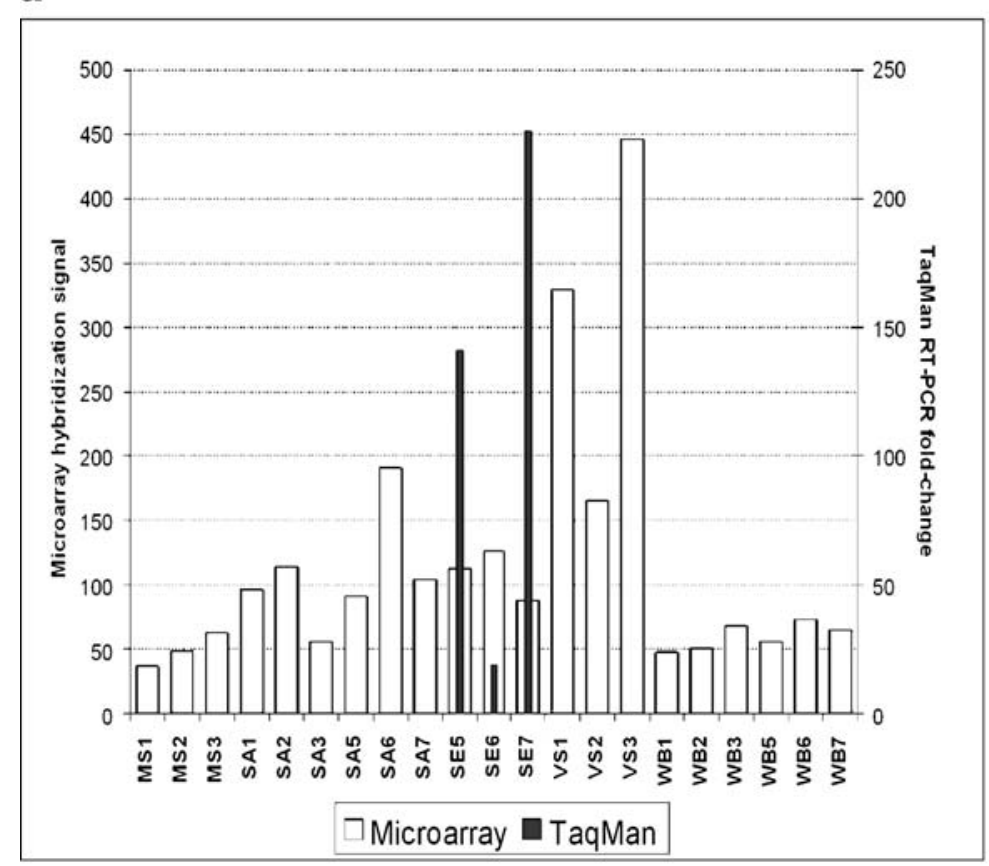

b

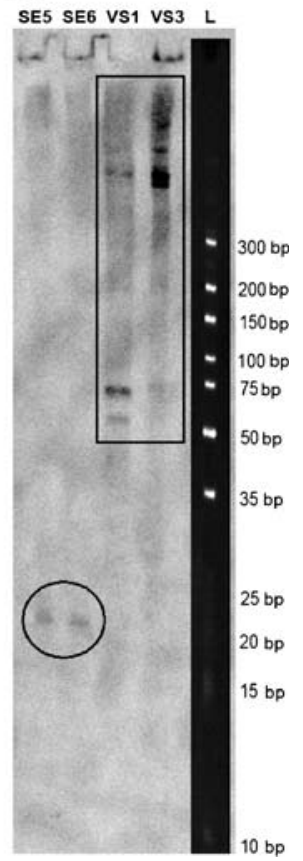

lighted here, only one appears human specific considering the available species data (miR-106a, which is not the most stringent blood marker we identified). Of the five semen markers highlighted, one is human specific (miR-891a, which is one of our most stringent semen markers), and two appear specific to human and non-human primates (miR-943 and miR-507). However, even if a miRNA marker itself is not human specific, the final conclusions retrievable from the approach we propose here for forensic application will still reveal human-specific information because the TaqMan assays of the three reference genes we used for normalization purposes are human specific.

\section{Conclusions}

Screening a large set of 718 human miRNA markers in all forensically relevant body fluids using the most comprehensive microarray platform currently available, we identified several RT-PCR confirmed miRNA markers that appeared over-expressed either in venous blood or in semen. We found that these blood and semen miRNA markers were practically not prone to degradation in samples stored for 1 year. Our results highlight four miRNA markers for blood identification (miR-20a, miR-106a, miR-185, and miR-144) and five for semen identification (miR-135a, miR10a, miR-507, miR-943, and miR-891a). Of those, two miRNA markers for blood (miR-144 and miR-185) and two others for semen (miR-135a and miR-891a) are suggestive to be most useful for body fluid identification in future forensic applications, and the respective RT-PCR assays used here for their detection were highly sensitive, allowing the reliable marker detection from subpicogram amounts of total RNA. Our results proved the applicability of the miRNA approach for forensic body fluids identification. However, despite that microarray analysis revealed distinct sets of differentially expressed miRNAs also for saliva, menstrual blood, and vaginal secretion, the microarray candidate markers for these three body fluids could not be confirmed by TaqMan-based RT-PCR. Hence, additional work remains to be carried out in search for miRNA markers for saliva, menstrual blood, and vaginal secretion identification and employing more suitable technology platform than applied here. Future work may also reveal the cell type of miRNA expression of the miRNA markers highlighted here, which may be relevant for certain forensic application, e.g., in case of semen markers, as well as additional marker details from dedicated forensic validation testing.

Acknowledgments We thank all volunteers for providing body fluid samples. We are grateful to Wilfred van IJcken (Center for Biomics, Erasmus University Medical Center Rotterdam) for the help in developing the miRNA microarray platform. This work was supported in part by funds from the Netherlands Forensic Institute, Fonds Schiedam-Vlaardingen, and received additional support by a grant from the Netherlands Genomics Initiative (NGI)/Netherlands Organization for Scientific Research (NWO) within the framework of the Forensic Genomics Consortium Netherlands (FGCN). 
Open Access This article is distributed under the terms of the Creative Commons Attribution Noncommercial License which permits any noncommercial use, distribution, and reproduction in any medium, provided the original author(s) and source are credited.

\section{References}

1. Bartel DP (2004) MicroRNAs: genomics, biogenesis, mechanism, and function. Cell 116:281-297

2. Rosenfeld N, Aharonov R, Meiri E, Rosenwald S, Spector Y, Zepeniuk M, Benjamin H, Shabes N, Tabak S, Levy A, Lebanony D, Goren Y et al (2008) MicroRNAs accurately identify cancer tissue origin. Nat Biotechnol 26:462-469

3. Sood P, Krek A, Zavolan M, Macino G, Rajewsky N (2006) Celltype-specific signatures of microRNAs on target mRNA expression. Proc Natl Acad Sci U S A 103:2746-2751

4. Liang Y, Ridzon D, Wong L, Chen C (2007) Characterization of microRNA expression profiles in normal human tissues. BMC Genomics 8:166

5. Kent EJ, Elliot DA, Miskelly GM (2003) Inhibition of bleachinduced luminol chemiluminescence. J Forensic Sci 48(1):64-67

6. Keating SM, Higgs DF (1994) The detection of amylase on swabs from sexual assault cases. J Forensic Sci Soc 34(2):89-93

7. Juusola J, Ballantyne J (2007) mRNA profiling for body fluid identification by multiplex quantitative RT-PCR. J Forensic Sci 52:1252-1262
8. Zubakov D, Hanekamp E, Kokshoorn M, van Ijcken W, Kayser M (2008) Stable RNA markers for identification of blood and saliva stains revealed from whole genome expression analysis of time-wise degraded samples. Int J Legal Med 122:135-142

9. Zubakov D, Kokshoorn M, Kloosterman A, Kayser M (2009) New markers for old stains: stable mRNA markers for blood and saliva identification from up to 16-year-old stains. Int J Legal Med 123:71-74

10. Pothof J, Verkaik NS, van IW W, EA Ta VT, van der Horst GT, Jaspers NG, van Gent DC, Hoeijmakers JH, Persengiev SP (2009) MicroRNA-mediated gene silencing modulates the UV-induced DNA-damage response. Embo J 28:2090-2099

11. Saeed AI, Sharov V, White J, Li J, Liang W, Bhagabati N, Braisted J, Klapa M, Currier T, Thiagarajan M, Sturn A, Snuffin M et al (2003) TM4: a free, open-source system for microarray data management and analysis. Biotechniques 34:374-378

12. Tibshirani R, Hastie T, Narasimhan B, Chu G (2002) Diagnosis of multiple cancer types by shrunken centroids of gene expression. Proc Natl Acad Sci U S A 99:6567-6572

13. Hanson EK, Lubenow H, Ballantyne J (2009) Identification of forensically relevant body fluids using a panel of differentially expressed microRNAs. Anal Biochem 387:303-314

14. Landgraf P, Rusu M, Sheridan R, Sewer A, Iovino N, Aravin A, Pfeffer S, Rice A, Kamphorst AO, Landthaler M, Lin C, Socci ND et al (2007) A mammalian microRNA expression atlas based on small RNA library sequencing. Cell 129:1401-1414 